\title{
Design of a High-Voltage Piezoelectric Converter for Airbag Ignition Modules
}

\author{
Hongbing Xiao ${ }^{\dagger}, \mathrm{Yu} \mathrm{Du}^{*}$, Chunyu Bai ${ }^{* *}$, Zerong Guo ${ }^{* * *}$, and Kang K. Yen ${ }^{* * * *}$ \\ ${ }^{\dagger}$ School of Computer and Information Engineering, Beijing Technology and Business University, Beijing, China \\ ${ }^{*}$ School of Mechanical, Electrical and Information Engineering, Shandong University, Weihai, China \\ ${ }^{* *}$ Beijing Changjiangyuan Science and Technology CO., LTD., Beijing, China \\ ${ }^{* * *}$ School of Mechatronics Engineering, Beijing Institute of Technology, Beijing, China \\ ${ }^{* * * *}$ Electrical and Computer Engineering, Florida International University, Miami, Florida, USA
}

\begin{abstract}
Due to the requirements for high reliability and accuracy, safety issues for airbag ignition systems need to be studied. In this paper, a high-voltage piezoelectric converter is designed to improve these requirements in airbag ignition systems. The proposed converter includes an inverter drive circuit, a Rosen piezoelectric transformer (PZT), an output circuit and a feedback control circuit. The key components of the high-voltage piezoelectric transformer are analyzed in detail. In addition, the proposed converter system is simulated and implemented for testing. The experimental results show that when the power supply is turned on, the charging time is less than $800 \mathrm{~ms}$. Furthermore, the output voltage of this converter can be kept between $2.9 \mathrm{kV}$ and $3.1 \mathrm{kV}$, under high-efficiency constant current charging mode and zero-voltage switching conditions.
\end{abstract}

Key words: Constant current charging, Electronic safety, High-voltage, Piezoelectric converter, Zero-voltage switching

\section{INTRODUCTION}

One of the most important parts of a vehicle to protect drivers and passengers is the airbag ignition system, which is shown in Fig.1. When an accident happens, the crash sensors send collision signals to a SRS-ECU (Supplemental Inflatable Restraint System, Electronic Controller Unit). Then the SRS-ECU enables the ignition module in the airbag system through an ignition voltage. Finally, the ignition module allows inflatable agents in the gas generator to release large amounts of nitrogen into the airbag, which instantly becomes lager to protect people in the vehicle. The major problem in existing airbag ignition modules is weak reliability caused by a low value of the ignition voltage, which is usually from 5 to $30 \mathrm{~V}$. Due to this problem, airbags cannot be reliably triggered. To make matters worse, airbags are sometimes

Manuscript received Oct. 9, 2012; revised Sep. 20, 2013

Recommended for publication by Associate Editor Tae-Woong Kim.

†Corresponding Author: xiaohbj@yahoo.com

Tel: +86-134-3952-9198, Beijing Technology and Business University

*School of Mechanical, Electrical and Information Engineering, Shandong University, China

${ }^{* *}$ Beijing Changjiangyuan Science and Technology CO., LTD., China

${ }^{* * *}$ School of Mechatronics Engineering, Beijing Institute of Tech., China

${ }^{* * * *}$ Electrical and Computer Engineering, Florida Int'l University, USA

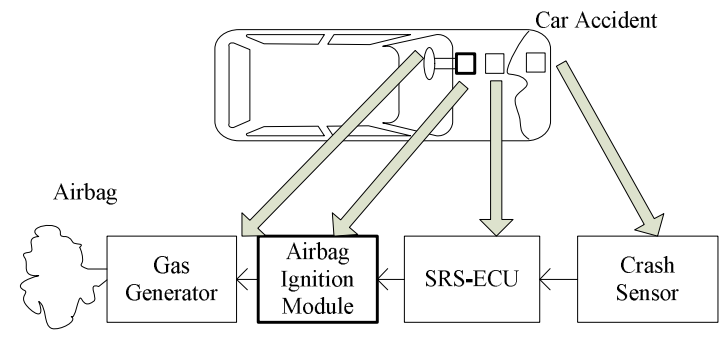

Fig.1. A schematic diagram of an airbag system.

triggered without an accident. This can occur due to electromagnetic interference in the car, because the electrostatic voltage sometimes reaches up to $1 \mathrm{kV}$ which can easily trigger the airbag ignition system. Thus, developing a high voltage converter is both necessary and essential. Furthermore, due to the particularity of automotive applications, the charging time for the high voltage converter should be very fast, and the high voltage converter should immediately work when the car is started. The proposed high-voltage converter system solves both problems with a high ignition voltage output from $2.9 \mathrm{kV}$ to $3.1 \mathrm{kV}$, and a fast charging time, which is less than $800 \mathrm{~ms}$.

Compared with traditional electromagnetic induction transformers, the piezoelectric transformer has the advantages 


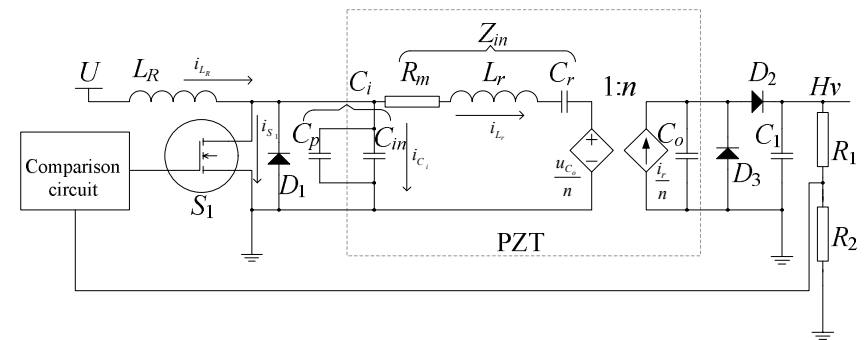

Fig.2. The piezoelectric high-voltage converter.

of a high power density, low electromagnetic noise, good electrical isolation of the input and output ports, a low cost, mass production capability, etc. However, PZTs have not been adopted in the design of automobile airbag ignition systems [1]. The current research on PZTs is focused on driving cold cathode fluorescent lamps, and PZTs have not been utilized in charging capacitors [2], [3]. The purpose of this study is to apply a PZT to the proposed high-voltage converter system in an airbag ignition system. The high-voltage output of the PZT at a proper operation frequency is used to improve the system stability and reliability under some constraints such as a limited supply voltage, a quick charge time (less than 1s) and a small size.

The switching drive frequency is a key parameter of the proposed high-voltage PZT converter system. The selection of this frequency directly affects the efficiency and the voltage gain of the PZT. In addition, this frequency should be able to drive a switch to achieve soft switching conditions for the high-voltage PZT converter to accomplish constant current charging (CCC). Currently, there are few studies on the charging power of the PZT [4], and a frequency analysis of the quantum control is not directly available in such fields. Although the quantum control mode [5] can facilitate the improvement of the system reliability, some constraints cannot be eliminated because of the driving frequency determination. In this paper, it is realized by both zero-voltage switching (ZVS) and CCC to optimize the choice of the drive frequency.

\section{Proposed High-Voltage Piezoelectric CONVERTER}

The proposed high-voltage piezoelectric converter system for electronic safety contains a main circuit and a quantum control circuit, as shown in Fig. 2. The main circuit includes an inverter drive circuit, a PZT and an output circuit. The inverter drive circuit has a switch circuit and an input matching circuit, while the output circuit consists of a rectifier circuit and a load circuit. In this figure, $Z_{i n}$ represents the impedance of the PZT including $R_{m}, L_{r}$ and $C_{r}$; while $C_{i n}$ is the input capacitance of the PZT and $C_{i}=C_{p} \| C_{i n}$. The feedback control circuit operates in the quantum control mode.

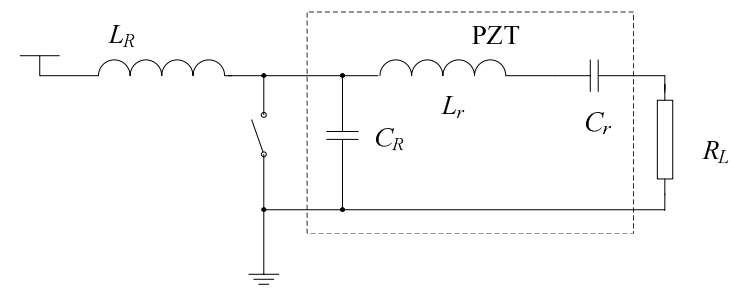

Fig.3. A simple switching converter circuit.

Through inverter switch $S_{1}$, a whole operation cycle includes one turning on and one turning off. When $S_{1}$ is turned on, the DC power charges the inductor $L_{R}$. When $S_{1}$ is off, the energy stored in the inductor discharges and begins to charge the PZT input capacitor. Then a quasi-sinusoidal voltage waveform is formed at the input of the PZT. The output voltage of the PZT is then raised up in turn. Through the doubler-voltage rectifier circuit, a pulse of DC current is generated to charge the high voltage capacitor $C_{1}$. A resistor divider sends the output voltage to the feedback control circuit. Afterwards a control action on switch $S_{1}$ is conducted through the comparison circuit module. If the control circuit detects the output level reaching a preset maximum, the converter drive circuit is turned off. If the opposite situation occurs i.e. the output level is lower than the preset value, the inverter drive circuit will work again. Through this process, the output voltage is controlled to within a preset range.

To drive switch $S_{1}$, an oscillator to generate a signal with a constant frequency and duty cycle is set for the switch. Since the switch input is typically a square wave signal, the output voltage is usually a sine wave or a quasi-sine wave signal through the inverter drive circuit. In the high-voltage converter circuit of an electronic safety system, the working voltage on the switch is several times that of the maximum DC power supply. The self-resonant frequency of the PZT is relatively high; and the switch in the inverter drive circuit must work in the soft-switching state in order to effectively reduce the switch power consumption. In addition, the inverter drive circuit should be flexible to adjust the output amplitude of the quasi-sinusoidal voltage, so as to adjust the output of the PZT to meet the design requirements. Because of the space constraint in this design, the converter adopts a single switch, which is shown in Fig. 3 together with the PZT.

The above circuit drives the PZT transformer with an inductor $L_{R}$ to achieve high efficiency. This inductor is connected in series to form a low-pass filter, which makes the input voltage waveform nearly sinusoidal. This enables the PZT transformer to operate in low-voltage stress, and removes the impact from high harmonics. It also reduces the loop current and the energy loss of the converter, and lets the power MOSFET inverter work in ZVS. In addition, the ZVS condition, which does not depend on the parameters of the PZT itself, can reduce the EMI noise produced by the converter on the system power source. 


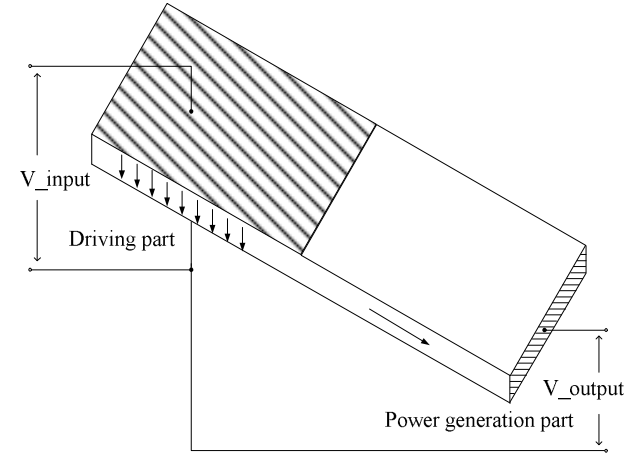

Fig.4. The Rosen-type PZT transformer.

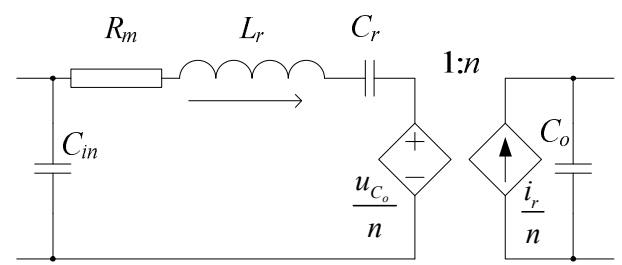

Fig.5. The improved equivalent circuit model of PZT.

Because of its high step-up ratio and larger output power, a Rosen-type PZT transformer is selected for the proposed design, as shown in Fig.4. The PZT consists of two parts: the input and the output, which are also known as the driving part and the power generation part, respectively. The driving part has a fired silver electrode along the thickness of the PZT, while the fired silver electrode of the power generation part is along the length of the PZT. The arrows in Fig.4 represent the direction of polarization in each part. For any PZT, the longer the length, the higher the step-up ratio. As a result, the length of the PZT should be made much longer than its thickness, and the output impedance and voltage should have larger values when compared with their input. Under normal circumstances, when the input voltage is in the range of several volts to tens of volts, the output voltage of the PZT is able to reach several thousand volts or more. In this design, up to $3.1 \mathrm{kV}$ of output voltage has been obtained.

In addition, the traditional ideal transformer model has been replaced by two independent controlled sources, as shown in Fig. 5. They are a controlled voltage source at the primary side and a controlled current source at the secondary side. Their parameters are either measured or calculated in Table I. Here, $C_{i n}$ stands for the input capacitor, $R_{m}$ denotes the leakage resistor, $C_{r}$ and $L_{r}$ are the resonant capacitor and inductor respectively, $C_{o}$ is the output capacitor, and $n$ signifies the transfer ratio.

In the DC high-voltage output of the PZT converter, the doubler-voltage rectifier circuit is the first choice for the PZT output. Doubler-voltage rectifier circuits are usually classified as an asymmetric topology with one or two capacitors or as a symmetrical half-bridge topology. Fig. 6 is a type of structures among them, which contains one
TABLE I

THE TRANSFORMER PARAMETERS

\begin{tabular}{|l|l|}
\hline$C_{i n}$ & $10.1 \mathrm{nF}$ \\
$R_{m}$ & $10.71 \Omega$ \\
$C_{r}$ & $301 \mathrm{pF}$ \\
$L_{r}$ & $15.12 \mathrm{mH}$ \\
$C_{o}$ & $18.37 \mathrm{pF}$ \\
$n$ & 10.25 \\
\hline
\end{tabular}

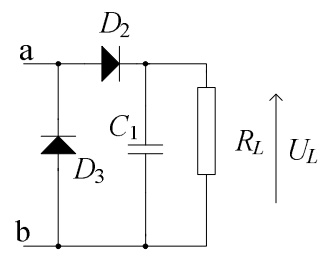

Fig.6. Topology of the doubler-voltage rectifier circuit.

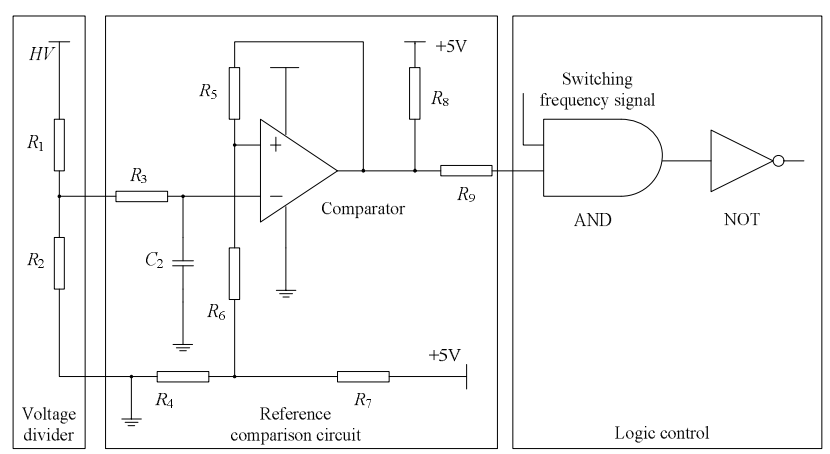

Fig.7. Comparison and logic control circuit.

capacitor $C_{1}$ and two diodes. It meets the system requirements simply in terms of the converter design.

The quantum control mode applies the converter output voltage as a feedback to control the circuit. This mode employs an oscillator to generate a drive signal with a constant frequency and duty cycle. Under the effect of this drive signal, the main circuit output level starts to rise. The control signal of the switching input is a high-frequency signal modulated by a low-frequency signal. The high-frequency signal is the selected switch drive signal, and the low-frequency signal is determined by both the upper and lower output voltage, and the discharge time constant. The PZT is able to filter out the low frequency signal which does not affect the performance of the converter. Under the loading short-circuit and no loading conditions, the converter can provide good self-protection. Therefore, no extra over-voltage or over-current protection circuits are required.

The comparison and logic control circuit is shown in Fig. 7. The sampled input signal from the output voltage enters into the comparator through the filter, and resistors $R_{4}-R_{7}$ are used to setup the upper and lower limits of the reference values. The logic control circuit is implemented by a NAND gate combined with a drive frequency signal. 


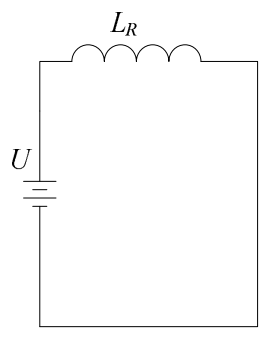

(a) $T_{\text {on }}$

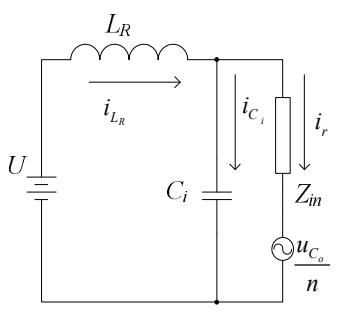

(b) $\mathrm{T}_{\text {off }}$
Fig.8. The equivalent circuit of an inverter driver.

\section{ANALYSIS AND DESIGN OF A High-VOLTAGE PIEZOELECTRIC CONVERTER}

\section{A. Analysis and Design of an Inverter Drive Circuit in the Zvs Condition}

As for the traditional switch, during the periods of on or off, the voltage and current waveforms across the switch overlap (neither $U$ nor $I$ is equal to zero), resulting in a switching loss $(P=U * I)$. The way to reduce this switching loss is to achieve soft switching. Through the resonance of the inductor and capacitor, the switch voltage (or current) will be a sinusoidal or quasi-sine wave. When the current crosses zero naturally, the switch is turned off, which is so-called ZCS (zero current switching). When the voltage falls to zero, the device is turned on, which is ZVS. Since $U=0$ or $I=0$, then $P=0$. Through the adjustments of $C_{\mathrm{i}}$ and $L_{\mathrm{R}}$ in Fig. 2, the voltage across switch $S_{1}$ can reach zero so as to operate in ZVS.

In accordance with the states of an inverter switch, an inverter drive circuit has two modes, mode 1 (switch on) and mode 2 (switch off). The equivalent circuit of the PZT primary part is shown in Fig. 8. The secondary part and the output circuit of the PZT will be discussed in B. Analysis and design of the charging circuit.

In mode 1 , when $t=t_{0}$, switch $S_{1}$ is on. Since the resistance of $S_{1}$ is small, the PZT is shorted and the DC power charges the inductor $L_{R}$ linearly. Assume that the resistances of the inductor and the switch-on can be ignored. Then the circuit in Mode 1 is described by:

$$
L_{R} \frac{d i_{L_{R}}}{d t}=U
$$

Therefore:

$$
i_{L_{R}}(t)=\frac{U}{L_{R}}\left(t-t_{0}\right)+i_{L_{R}}\left(t_{0}\right)
$$

In mode 2, when $t=t_{1}$, switch $S_{1}$ is off. As a result, the inductor discharges and begins to charge the input capacitor of the PZT to form a resonance voltage. In order to obtain the ideal condition of ZVS, the selected switch drive frequency should be slightly larger than the operating frequency of the PZT. As a result, the resonant branch impedance of the PZT shows inductance characteristics. Meanwhile, the capacitance $C_{i}$ of the PZT provides a lower impedance path, so that the inductor $L_{R}$ and the capacitor $C_{i}$ are the major resonant components for the inverter. There are the following relationships in Mode 2:

$$
\begin{gathered}
L_{R} \frac{d i_{L_{R}}}{d t}+U_{c_{i}}=U \\
C_{i} \frac{d u_{c_{i}}}{d t}=i_{c_{i}} \\
i_{L_{R}}=i_{C_{i}}
\end{gathered}
$$

Combining (3)-(5), results in:

$$
\begin{gathered}
U_{S_{1}}(t)=Z \bullet i_{L R}\left(t_{1}\right) \sin \omega_{0}\left(t-t_{1}\right)+\left[1-\cos \omega_{0}\left(t-t_{1}\right)\right] U \\
i_{L R}(t)=\frac{U}{Z} \sin \omega_{0}\left(t-t_{1}\right)+i_{L R}\left(t_{1}\right) \cos \omega_{0}\left(t-t_{1}\right)
\end{gathered}
$$

in which:

$$
\omega_{0}=\frac{1}{\sqrt{L_{R} C_{i}}}, Z=\sqrt{\frac{L_{R}}{C_{i}}}, u_{S_{1}}=u_{c_{i}}
$$

Equation (6) shows that the peak voltage of switch $S_{1}$ changes with the size of the duty cycle $D$ in the case of the given PZT and the resonant inductor. To reduce the voltage stress on the switch, the value of $D$ should not be too large. Actually, $D$ should be determined by a compromise between the transformer step-up ratio, the output voltage requirement and the withstanding voltage of the switch.

The inductor $L_{R}$ and the capacitor $C_{i}$ of the transformer play the role of a low-pass filter and provide an effective way for the soft-switching mode. By adjusting the value of the inductor $L_{R}$, the capacitor $C_{i}$, and the drive signal duty cycle, the flexibility to adjust the input voltage of the transformer is achieved.

The authors of [6] proposed that the resonant circuit frequency, which is formed by the inductor $L_{R}$ and the input capacitor $C_{i n}$ of the PZT, should be as close as possible to the work frequency:

$$
f_{w}=\frac{1}{2 \pi \sqrt{L_{R} C_{i n}}}
$$

However, for the drive signals of any duty cycle, the values of the inductor $L_{R}$ by this formula are not well suited to accomplish the ZVS condition. In addition, the input voltage of the transformer cannot provide flexible regulation. Combining Equation (6) with the requirement of the ZVS condition, the inverter driver parameters should be selected so that the voltage $u_{S 1}$ is zero in the whole low level of the drive signal $u_{G S}$. Then the following relationship is available:

$$
\left[\pi+2 \arctan \frac{U}{Z \bullet i\left(t_{1}\right)}\right] \sqrt{L_{R} C_{i}} \leq \frac{1}{f_{d}}(1-D)
$$

where $f_{d}$ is the drive frequency.

Here, $C_{i n}$ is substituted by $C_{i}\left(C_{i n}, C_{p}\right)$. The parameters $L_{R}$, $C_{i}$, and the duty cycle $D$ are determined after obtaining the maximum voltage of the transformer input and the drive signal frequency by the combination of Equations (6) and (9). As a result, the proposed scheme is able to achieve the 


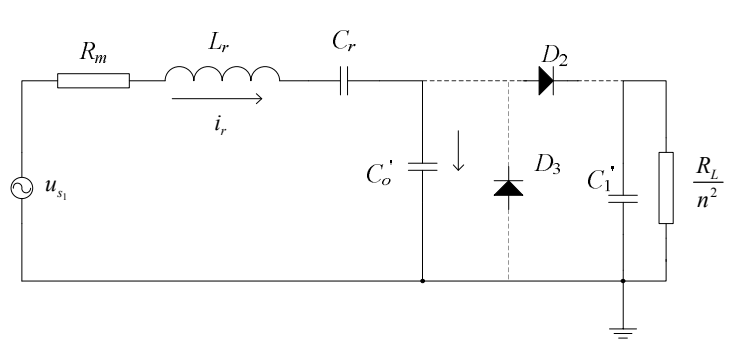

(a)

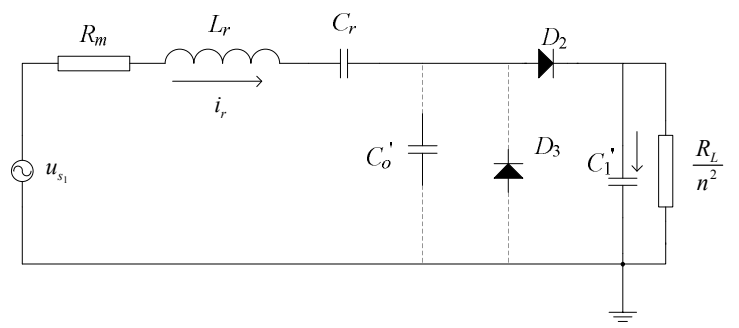

(b)

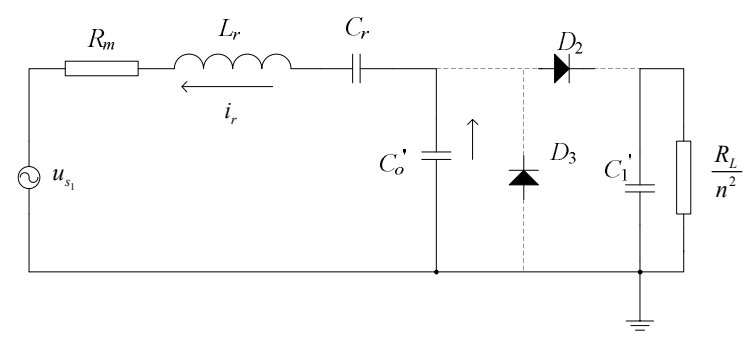

(c)

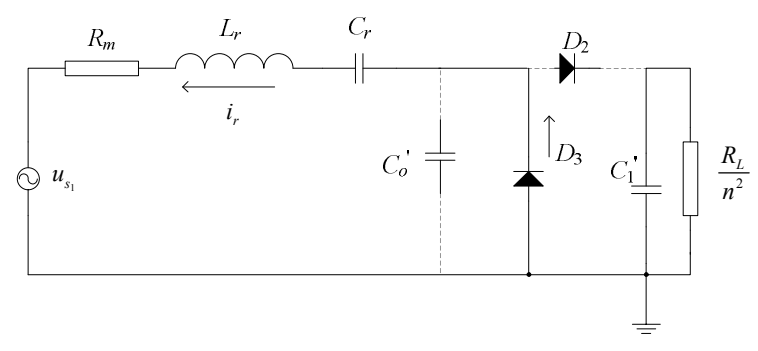

(d)

Fig.9. The equivalent circuit of the converter working states.

flexible adjustment of the input voltage of the transformer, and realize the ZVS condition.

\section{B. Analysis and Design of the Charging Circuit}

Unlike the analysis of general series and parallel resonant converters, the voltage of a charged capacitor is a variable. During the charging time, the output voltage gradually increases. Suppose that the circuit components work in the ideal state, and the full charging cycle of the converter is divided into four states. The output capacitance of the secondary of the PZT, $C_{o}$, is mapped to the primary and it is denoted as $C_{o}^{\prime}$, and the loaded high voltage capacitance $C_{1}$ is mapped as $C_{1}^{\prime}$. The state analysis for the equivalent circuit is as follows, where $C_{o}^{\prime}=n^{2} C_{o}$, and $C_{1}^{\prime}=n^{2} C_{1}$.

State $1\left[t_{0}-t_{1}\right]$ : In order to facilitate the analysis, the zero

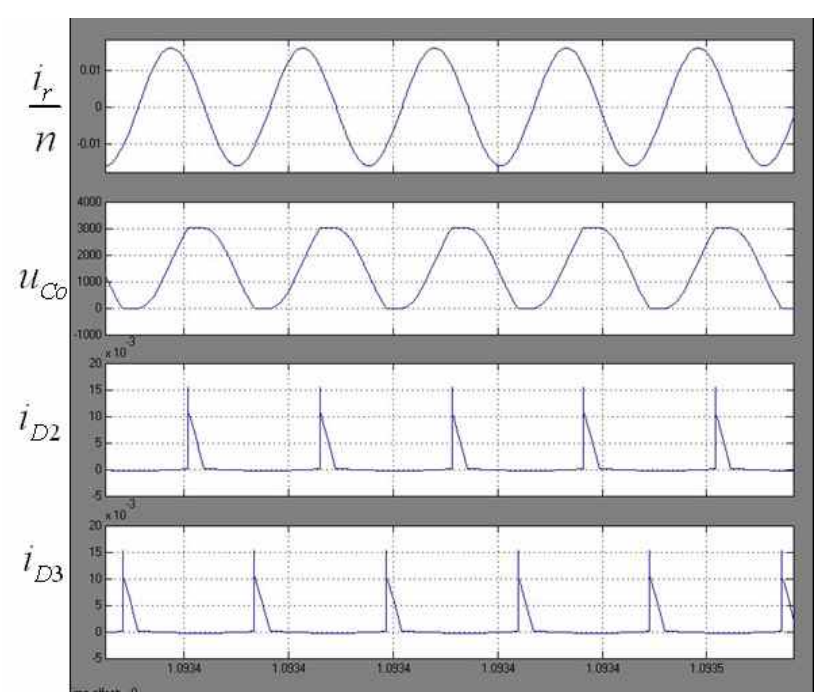

Fig.10. Current and voltage waveforms of doubler-voltage rectifier circuit.

point of the positive current is regarded as the time zero point $t_{0}$, and the equivalent circuit is illustrated in Fig. 9 (a). At $t_{0}$, the resonant current $i_{r}$ starts to charge the capacitor $C_{o}^{\prime}$ from zero. Then it generates a sine wave in resonance, which means the circuit is in the internal resonant state and does not provide energy to the load. When the voltage of $C_{o}^{\prime}$ rises to $U_{c_{p}}=U_{L} / n$, this state ends.

State $2\left[t_{1}-t_{2}\right]$ : At $t_{1}$, diode $D_{2}$ is turned on, and the circuit begins to generate energy to the load. At this time, $C_{o}^{\prime}$ quits the resonance and $C_{1}^{\prime}$ takes part in the resonant circuit. Usually $C_{1}^{\prime} \ll C_{r}$, and the resonant characteristic of the circuit is almost free from the influence of $C_{1}^{\prime}$. When the positive resonant current is reduced to zero, this state ends. Fig. 9 (b) illustrates the equivalent circuit of this state.

State $3\left[t_{2}-t_{3}\right]$ : At $t_{2}$, the positive resonant current reduces to zero and $D_{2}$ is turned off. Then $C_{o}^{\prime}$ joins the resonant circuit. The circuit stops sending energy to the load and works in the internal resonance. The voltage of $C_{o}^{\prime}$ begins to decline. When it reaches zero, this state ends. Fig. 9 (c) shows the equivalent circuit of this state.

State $4\left[t_{3}-t_{4}\right]$ : At $t_{3}$, diode $D_{3}$ is switched on, and $C_{o}^{\prime}$ quits the resonance. This state is completed and begins to return to state 1 . This means that an entire cycle has finished. Fig. 9 (d) illustrates the equivalent circuit for this state.

The current $i_{r} / n$ flows into the transformer output capacitor $C_{o}$ when the two rectifier diodes are in their periods of non-conducting. Mainly, there are two rectifier diode conduction periods, and the voltage of the output capacitor $C_{o}$ is equal to the high-voltage of the load in the $D_{2}$ period and is zero in the $D_{3}$ period. The related current and voltage waveforms are shown in Fig. 10. 
When the voltage of the output capacitor $C_{o}$ for the PZT is in the diode non-conduction period, it is divided into interval functions of Equation (10) according to the phase intervals and initial values [7].

$u_{c_{o}}=\left\{\begin{array}{cc}\frac{U_{L}}{1+\cos \theta}(1-\cos \vartheta) & 0<\vartheta<\pi-\theta \\ U_{L} & \pi-\theta<\vartheta<\pi \\ \frac{U_{L}}{1+\cos \theta}(\cos \theta-\cos \vartheta) & \pi<\vartheta<2 \pi-\theta \\ 0 & 2 \pi-\theta<\vartheta<2 \pi\end{array}\right.$

where $U_{L}$ is the voltage of the load, $\theta$ is the conduction angle, and $\vartheta$ is the phase.

It is shown that the CCC mode is able to maximize the capacitor charge efficiency, which is an important quality standard for evaluating capacitor charging performance. The next process is a detailed analysis for the converter to achieve the $\mathrm{CCC}$ condition.

The charging power supply of the capacitor is different from the normal charging process of a DC power supply. The former output voltage steps up with increments of the duty cycles. Here the corresponding diode conduction angle, the maximum resonant current and the initial output voltage of the $m^{\text {th }}$ working cycle are expressed as $\theta_{m}, I_{r m(m)}$ and $U_{L(m-1)}$, respectively. Equation (10) gives:

$$
\frac{I_{r m(m)}}{n}=\frac{U_{L(m-1)} \omega C_{o}}{1+\cos \theta_{m}}
$$

where $\omega=2 \pi f$. Thus, the average current through diode $D_{2}$ at any $m^{\text {th }}$ working cycle is:

$$
\begin{gathered}
I_{D 2 a v}=\frac{1}{2 \pi} \int_{\pi-\theta_{m}}^{\pi} \frac{I_{r m(m)}}{n} \sin \vartheta d \vartheta \\
=\frac{1}{2 \pi} \frac{I_{r m(m)}}{n}\left(1-\cos \theta_{m}\right) \\
=\frac{1}{2 \pi} U_{L(m-1)} \omega C_{o} \tan ^{2}\left(\frac{\theta_{m}}{2}\right)
\end{gathered}
$$

In this cycle, the quantity of charging is:

$$
Q_{m}=I_{D 2 a v} \cdot \frac{2 \pi}{\omega}
$$

Assuming the linear CCC condition of the capacitor and ignoring the energy consumption by the load resistor divider, the quantity of the charging in the $m^{\text {th }}$ cycle is equal to the $(m-1)^{\text {th }}$ cycle:

$$
Q_{m}=Q_{m+1}
$$

Substituting Equations (12) and (13) into (14), yields:

$$
U_{L(m-1)} C_{o} \tan ^{2}\left(\frac{\theta_{m}}{2}\right)=U_{L(m)} C_{o} \tan ^{2}\left(\frac{\theta_{m+1}}{2}\right)
$$

And

$$
U_{L(m)}=U_{L(m-1)}+Q_{m} / C_{1}
$$

From equations (15) and (16), the recurrence formula of

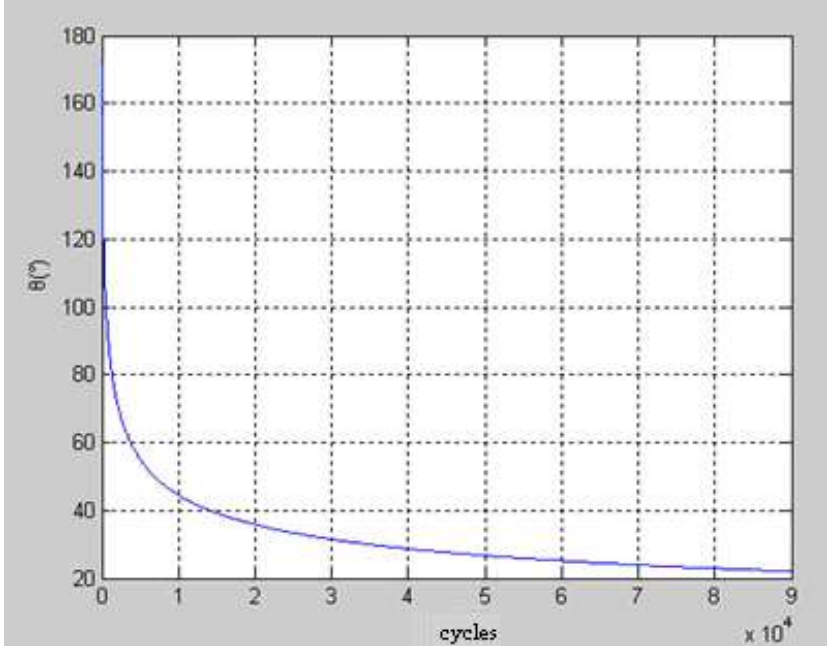

Fig.11. Relationship between conduction angle and cycles.

the conduction angle change in the CCC condition is [8]:

$$
\tan ^{2}\left(\frac{\theta_{m+1}}{2}\right)=\frac{\tan ^{2}\left(\frac{\theta_{m}}{2}\right)}{1+\frac{C_{o}}{C_{1}} \cdot \tan ^{2}\left(\frac{\theta_{m}}{2}\right)}
$$

The relationship between the calculated conduction angle (using the numerical analysis by equation (17)) and the number of the operation cycles is shown in Fig. 11. Here, the initial value of $\theta$ is $\pi$. It can be seen from the graph that with an increase of the charge cycles, the conduction angle decreases. At around 5,000 charge cycles, the conduction angle drops rapidly and after that it gradually slows down. Finally the conduction angle reaches a stable value.

Equation (11) shows that the resonant currents of the $(m+1)^{\text {th }}$ and $m^{\text {th }}$ working cycles meet the relationship:

$$
\frac{I_{r m(m+1)}}{I_{r m(m)}}=\frac{\left(1+\cos \theta_{m+1}\right) U_{L(m)}}{\left(1+\cos \theta_{m}\right) U_{L(m-1)}}
$$

When the constant-current charging in equal-step is achieved by the high-voltage capacitor of the converter, it is clear that:

$$
\begin{aligned}
& U_{L(m)}=m \cdot \Delta U \\
& U_{L(m+1)}=(m+1) \Delta U
\end{aligned}
$$

Therefore, from equation (18), the maximum resonant current should satisfy the following equation to achieve the CCC condition:

$$
\frac{I_{r m(m+1)}}{I_{r m(m)}}=\frac{m}{m-1}
$$

This denotes $\Delta I_{r m(m)}=I_{r m(m+1)}-I_{r m(m)}$, then:

$$
\Delta I_{r m(m)}=I_{r m(m+1)} / m
$$

and:

$$
I_{r m(m+1)}=m \times \Delta I_{r m(m)}
$$

Equation (22) shows that when the maximum of resonant 


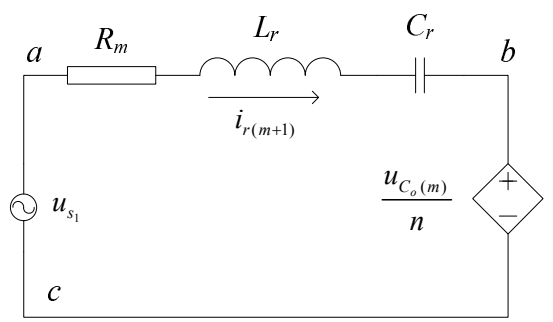

Fig.12. The primary side equivalent circuit of the PZT.

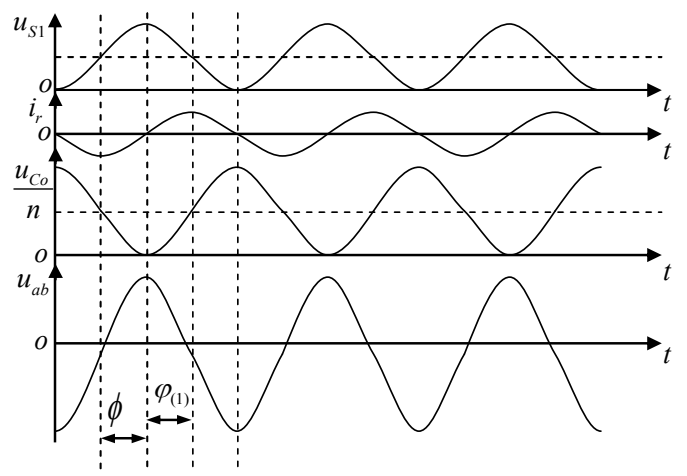

Fig.13. The main waves of the resonant branch.

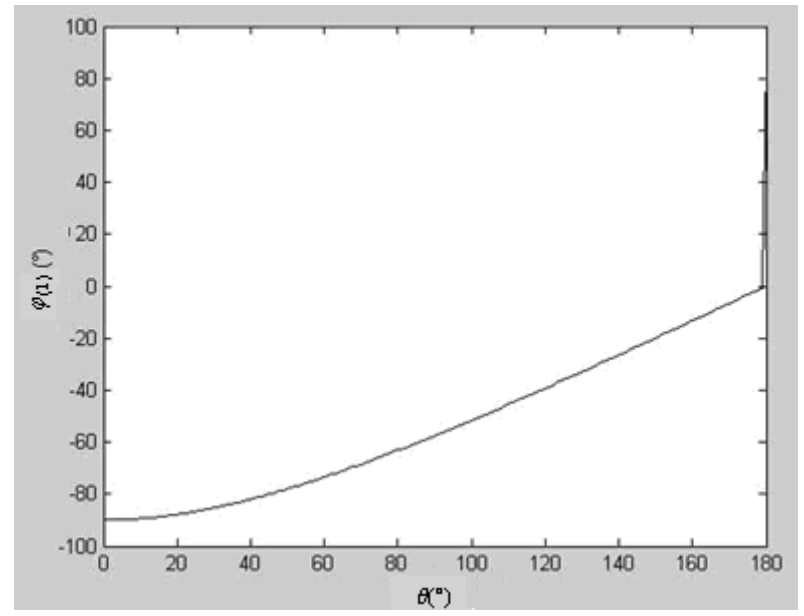

Fig.14. Relationship between $\varphi_{1}$ and $\theta$.

current is small at the beginning and increases in equal-step, the capacitor of the converter is approximately charged in equal-step.

\section{Analysis and Design of the Drive Frequency}

Considering the primary side of the PZT in the converter circuit, the equivalent circuit is shown in Fig. 12. When the load capacitor is charged by the converter, the main curves are presented in Fig. 13.

In the fixed-frequency control mode, the key consideration in the charging circuit is the late stage of charging. In the latter part of the output capacitor charging procedure, the phase of the voltage fundamental wave is a quarter cycle lag when compared with the phase of the resonant current, which is shown as $\varphi_{(1)}$ in Fig. 14.
At the selected frequency, the impedance between nodes $a$ and $b$ in Fig. 12 is definite. Combining this property with the equal-step charging condition given by Equation (20), the proposed system is able to approximately meet the CCC condition, as long as the maximum value of $V_{a b}$ is increased in equal-step. Fig. 13 shows the phase differences among $u_{S 1}$, $i_{r}$ and $u_{C o} / n$ at the same time. If the phase difference between the resonant current and the input voltage is about $90^{\circ}$, as shown in Equation (23), it is capable of meeting the requirements of the above analysis.

$$
\arctan \left(\frac{R_{m}}{\sqrt{\left(L_{r} \omega_{s}-\frac{1}{C_{r} \omega_{s}}\right)^{2}}}\right)=\phi \rightarrow 90^{\circ}
$$

where $\omega_{s}$ is the serial resonant frequency.

As a result, the corresponding drive frequency is:

$$
f_{d}=\frac{\omega_{s}}{2 \pi}
$$

At this time, $u_{S 1}$ and $u_{C o(1)} / n$ have the reverse phase. Therefore, their peak-to-peak voltage can be a maximum value. Because of the increasing trend of $u_{C o(1)} / n$, the peak-to-peak value of $u_{a b}$ rises in equal-step as follows:

$$
u_{a b(p-p)}=u_{s 1(p-p)}+\frac{U_{C o_{(1)}(p-p)}}{n}
$$

The corresponding peak-to-peak value of the resonant current is:

$$
i_{r(p-p)}=\frac{u_{a b(p-p)}}{\sqrt{R_{m}^{2}+\left(L_{r} \omega_{s}-\frac{1}{C_{r} \omega_{s}}\right)^{2}}}
$$

It shows that when the charge cycles increase, the conduction angle decreases in the equal-step charging condition. The durations for state 1 and state 3 enlarge progressively while the durations for state 2 and state 4 decline step by step. When the equivalent circuit works in the resonant state, the resonant circuit gets the maximum voltage gain and current values.

The operating frequency for states 2 and 4 are:

$$
f_{w 2,4}^{\prime}=\frac{1}{2 \pi \sqrt{L_{r} C_{r}}}
$$

The operating frequency for states 1 and 3 are:

$$
f_{w 1,3}^{\prime}=\frac{1}{2 \pi \sqrt{L_{r} \cdot \frac{C_{r} \cdot n^{2} C_{o}}{C_{r}+n^{2} C_{o}}}}
$$

Equation (28) gives the upper limit of the drive frequency, and this value meets a large step-up ratio in the late state of the charging. Meanwhile, $f_{\mathrm{w} 1,3}$ is larger than the actual operating value. Thus, this frequency is able to realize soft-switching to create a good work condition.

Substituting the parameter values of the selected transformer into Equations (24) and (27-28) with a selected $100 \mathrm{M} \Omega$ sampling divider resistance, the frequency values for 
$f_{d}, f_{\mathrm{w} 2,4}^{\prime}$, and $f_{\mathrm{w} 1,3}^{\prime}$ are $79.5 \mathrm{kHz}, 74.6 \mathrm{kHz}$, and $80.2 \mathrm{kHz}$, respectively. In all, the switch drive frequencies are analyzed and calculated from different perspectives, and the results indicate consistency.

\section{Design of the Quantum Control Circuit}

The quantum control circuit mainly consists of a voltage sampling divider circuit, a reference comparison circuit and a logic control circuit. The sampling circuit obtains the output voltage from a high-voltage capacitor and sends it to the reference comparison circuit. The comparison circuit compares the output voltage to the upper and lower limits of the preset reference voltage and then exports a corresponding high or low level. Together with the comparison of the circuit output signal, the logic control circuit is responsible for generating a selected switching-frequency with a fixed duty cycle. As a result, the modulated output is applied to drive the switch.

The design principle of the sampling resistor divider is to guarantee the capacitor charging efficiency, without affecting the operation characteristics of the PZT. The output power is:

$$
\begin{aligned}
& P_{L m}=\frac{\left(U_{C o(1) \max }\right)^{2}}{2 R_{L e q}} \\
& =\frac{U_{L(m)}^{2}}{R_{L}}+\left(\frac{1}{2 T_{d}} C_{1} U_{L(m)}^{2}-\frac{1}{2 T_{d}} C_{1}\left(U_{L(m)}-\frac{U_{L(m)}}{m}\right)^{2}\right) \\
& =\frac{U^{2}{ }_{L(m)}}{R_{L}}+\left(\frac{1}{2} f_{d} C_{1} U_{L(m)}^{2}-\frac{1}{2} f_{d} C_{1}\left(U_{L(m)}-\frac{U_{L(m)}}{m}\right)^{2}\right)
\end{aligned}
$$

From the above equation, the converter's output impedance is deduced as:

$$
R_{\text {Leq }}=\frac{k_{u(1)}^{2} R_{L}}{8+4 R_{L} C_{1} f_{d}\left(\frac{2 m-1}{m^{2}}\right)}
$$

where $f_{d}$ is the drive frequency, and $k_{u(1)}$ indicates the voltage waveform coefficient [8]. Without a sampling resistor in the converter output, the output power becomes:

$$
\begin{gathered}
P_{L_{m}}=\frac{\left(u_{C o(1) \max }\right)^{2}}{2 R_{L e q}^{\prime}}=\frac{1}{2} f_{d} C_{1} U_{L(m)}^{2}-\frac{1}{2} f_{d} C_{1}\left(U_{L(m)}-\frac{U_{L(m)}}{m}\right)^{2} \\
P_{L_{m}}=\frac{\left(U_{C o(1) \max }\right)^{2}}{2 R_{L e q}^{\prime}} \\
=\frac{1}{2} f_{d} C_{1} U_{L(m)}^{2}-\frac{1}{2} f_{d} C_{1}\left(U_{L(m)}-\frac{U_{L(m)}}{m}\right)^{2}
\end{gathered}
$$

Therefore:

$$
\frac{R_{\text {Leq }}^{\prime}}{R_{\text {Leq }}}=\frac{2}{R_{L} f_{d} C_{1}\left(\frac{2 m-1}{m^{2}}\right)}+1
$$

The power consumption of the voltage sampling resistor should be much less than the converter output power, and the impedance change of the resistor should not affect the transformer's frequency characteristics in the early charging.

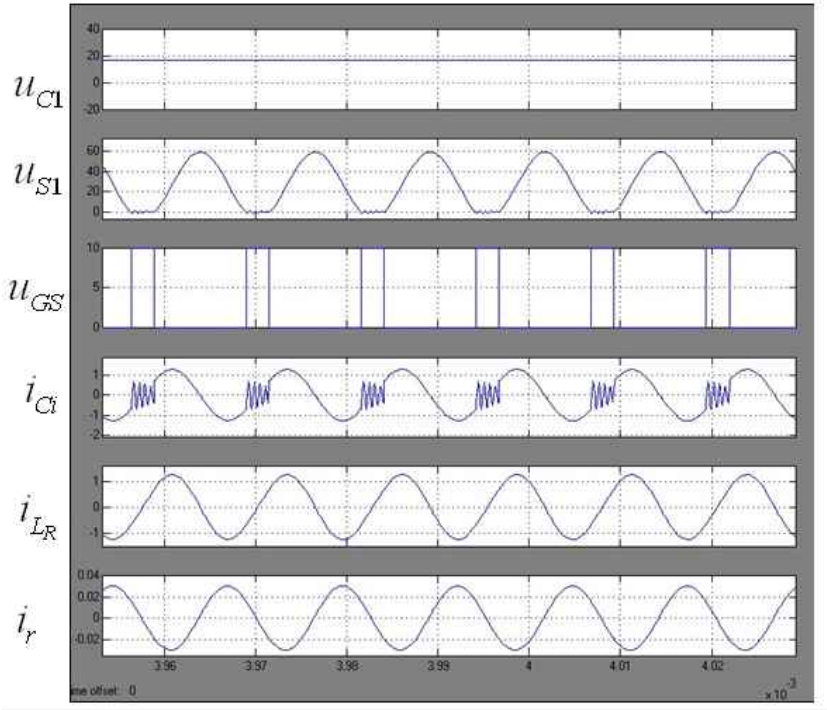

(a)

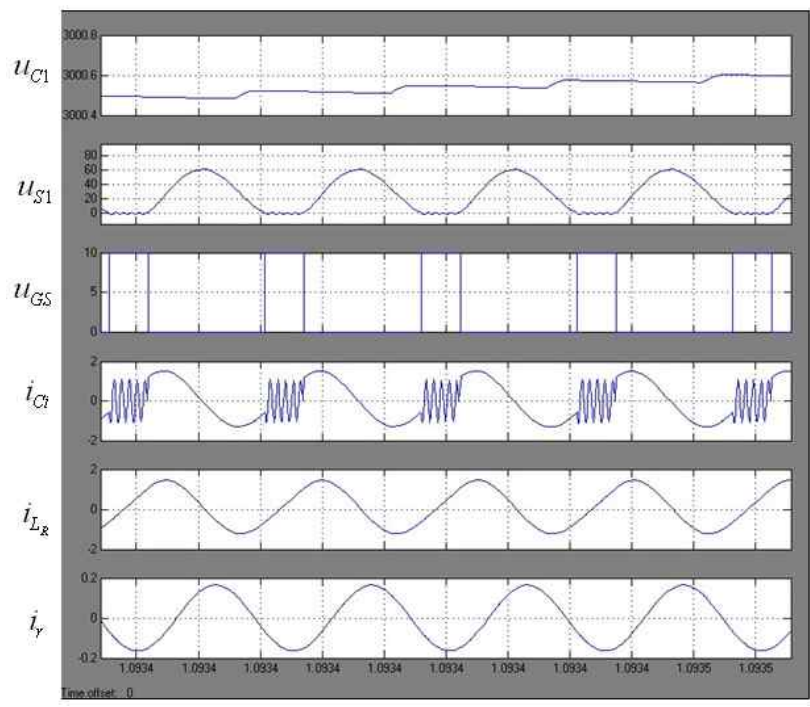

(b)

Fig.15. The main output voltage waveforms.

Moreover, it should ensure that the difference between the equivalent resistances is no more than one order of the magnitude. From Equation (32), $R_{L}=100 \mathrm{M} \Omega$ meets this requirement.

\section{SIMULATION}

A circuit simulation is carried out by applying MATLAB's SimPowerSystems Tool Box [9]. With the components and related modules provided by the Box, the simulation is performed in strict accordance with the electrical connections of the actual circuit in the modeling. In this way, the simulation model becomes visual and it is relatively simple to adjust the values of the circuit parameters. Fig. 15(a) and (b) present the simulation results of the main associated signal output voltage waveforms. They clearly show that all of the circuit waveforms are consistent with the analysis results. These simulation results verify the correctness of the above 
TABLE II

THE EXPERIMENTAL CONFIGURATION AND RESUlts

\begin{tabular}{|l|l|}
\hline Components & Parameteres/Sizes \\
\hline$S_{I}$ & FET IRLR3410 \\
$C_{I}$ & $0.22 \mu \mathrm{F} / 4 \mathrm{kV}$ \\
$D_{I}$ & GL506 \\
$R_{I}$ & $100 \mathrm{~K} \Omega$ \\
$R_{2}$ & $100 \mathrm{M} \Omega$ \\
$D$ & 0.2 \\
$L_{R}$ & $47 \mu \mathrm{H}$ \\
$C_{P}$ & $78 \mathrm{nF}$ \\
Drive signal frequency & $79.4 \mathrm{kHz}$ \\
Input DC voltage & $28 \mathrm{~V}$ \\
\hline Output & Experimental results \\
\hline$u_{S 1}$ & $58 \mathrm{~V}$ \\
$u_{C l}$ & $3.0 \mathrm{kV}$ \\
\hline
\end{tabular}

analysis.

Figures 15 (a) and (b) demonstrate the early charging and late charging wave curves, respectively. They show that the output voltage waveform of the high-voltage capacitor $u_{C 1}$ increases in equal step. Here, the voltage waveform $u_{s 1}$ is a quasi-sine. It starts from zero when the switch is closed, and the voltage decreases exactly to zero when the switch is opened $\left(u_{G S}\right.$ is at a high level). The switch operates in the soft-switching mode, which significantly reduces switching losses. Meanwhile, the maximum input voltage $u_{s 1}$ is $60 \mathrm{~V}$, which is consistent with the measured value of the circuit. It can be seen that the capacitance-voltage curve increases in equal-step in the quantum control mode during the process of output voltage change, which indicates that the high voltage capacitor charging circuit works in a highly efficient CCC mode.

\section{EXPERIMENTAL RESULTS}

In order to verify the correctness of the proposed converter circuit, a prototype was developed. The parameters of the main circuit components and the experimental results of the prototype converter are shown in TABLE II.

It is mentioned that the calculated value of the drive frequency should be slightly larger than the measurement value. Therefore, the calculated frequency is selected directly, and is able to achieve the ZVS condition.

Experimental waveforms of the drive signal voltage $u_{G S}$ and the transformer input voltage $u_{S 1}$ are shown in Fig. 16. Here the input DC voltage, the drive signal frequency and the duty cycle $D$ are set to $28 \mathrm{~V}, 79.4 \mathrm{kHz}$ and 0.2 , respectively. The inductance of $L_{R}$ is calculated as $47 \mu \mathrm{H}$. The parallel input capacitance $C_{P}$ is set to $78 \mathrm{nF}$.

It can be seen that the inverter switch actually works in the ZVS mode and that the maximum of $u_{S 1}$ is approximately $58 \mathrm{~V}$, which is in accordance with the calculated result from Equation (6).

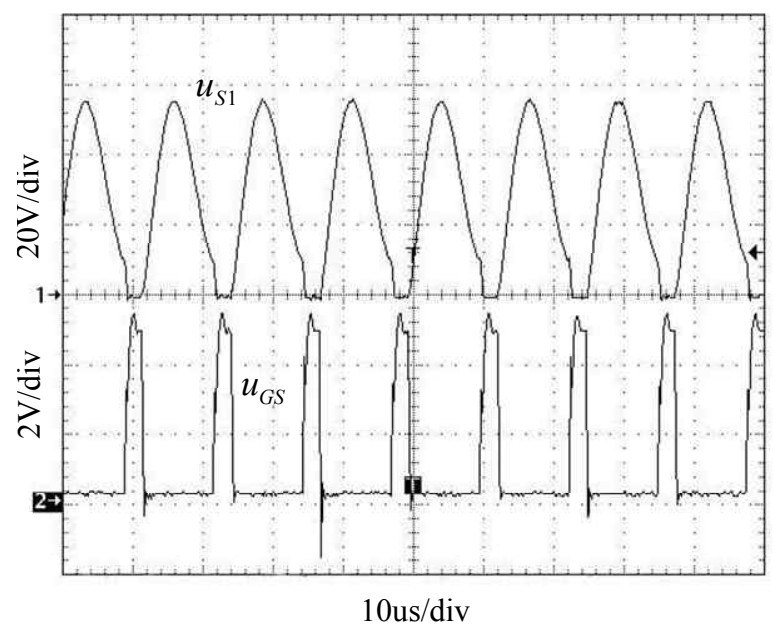

Fig.16. Experiment waveforms of $u_{\mathrm{GS}}$ and $u_{S 1}$

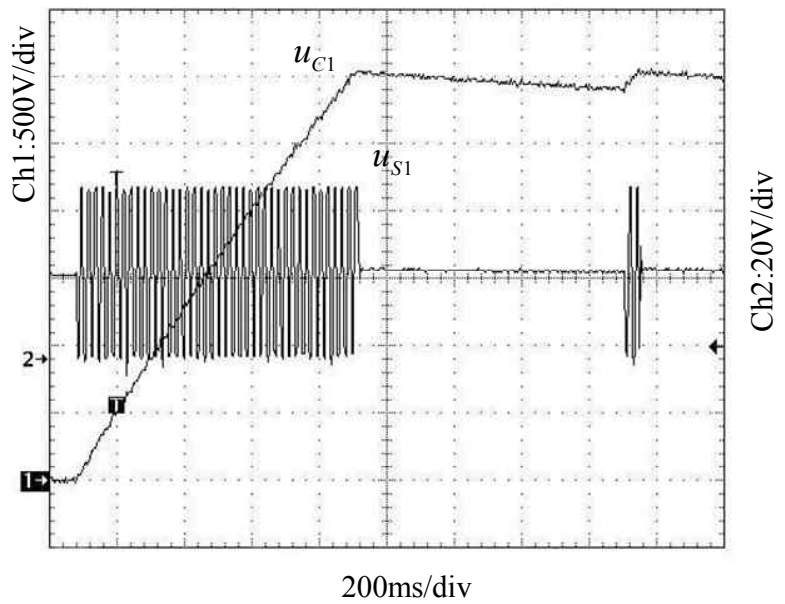

Fig.17. Experiment waveforms of $u_{S 1}$ and $u_{\mathrm{c} 1}$.

At room temperature, the waveforms of the input voltage and the output voltage of the load capacitance are indicated in Fig. 17. The output voltage is controlled at a range from $2.9 \mathrm{kV}$ to $3.1 \mathrm{kV}$ in less than $800 \mathrm{~ms}$. It is obvious that the charging time from the power-on is quick enough for an airbag to be triggered. This shows that in the quantum control mode, the ascending slope of the capacitance-voltage curve is almost constant in the changing process of the output voltage. This indicates that the high voltage capacitor charges in a highly efficient CCC mode.

Waveforms of the PZT resonant branch current $i_{r}$ and the output voltage $u_{c 1}$ of the load capacitance are shown in Fig. 18. In the late charging process, the envelope curve of the resonant current trend is in accordance with the equal-step output voltage, which verifies the CCC mode. The waveform of the high voltage capacitor in the discharge of a short-circuit is illustrated in Fig. 19. The sharp decline in the curve shows that the circuit is able to continue to work under a load discharge. If there is no-load, the voltage is still controlled at the preset range. 


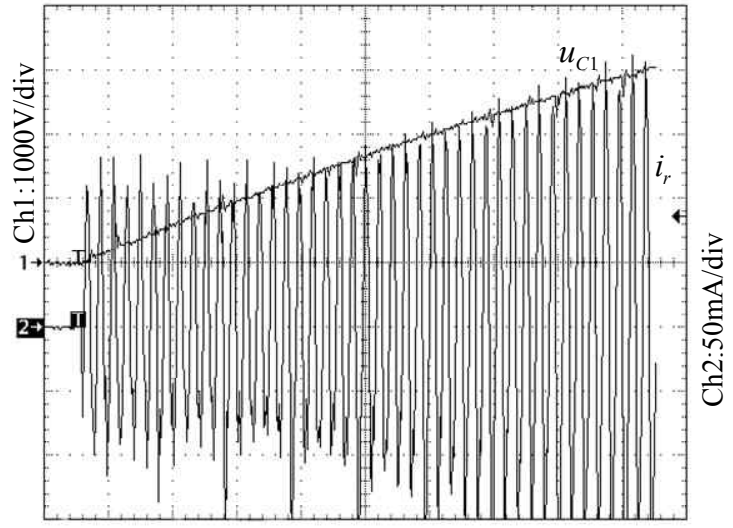

$100 \mathrm{~ms} / \mathrm{div}$

Fig.18. Experiment waveforms of $i_{\mathrm{r}}$ and $u_{\mathrm{cl}}$.

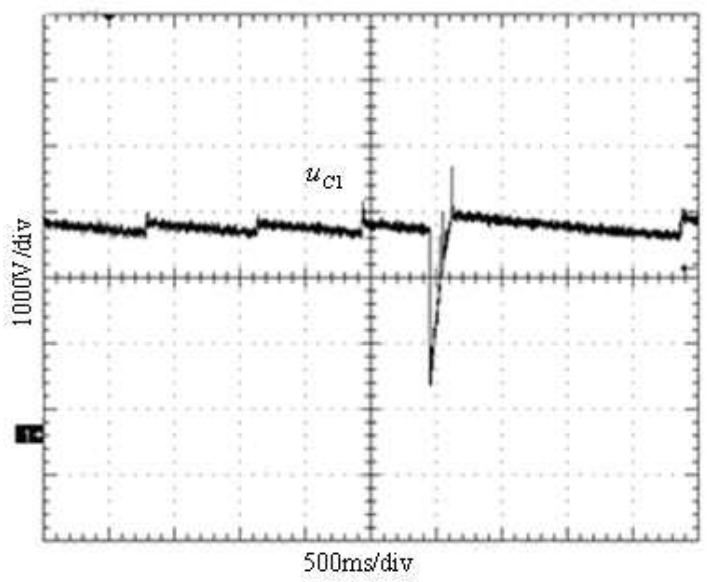

Fig.19. The high voltage capacitor waveform in load short-circuit discharge.

The designed PZT converter has been utilized in an airbag safety ignition system, and the test PCB is shown in Fig. 20. When compared with current airbag ignition systems, the proposed system has a smaller size and less weight because a PZT is utilized. In addition, higher reliability and accuracy are gained through its $2.9 \mathrm{kV}$ to $3.1 \mathrm{kV}$ ignition energy in a highly efficient mode. When a collision occurs, the high-voltage capacitor triggers the airbag more quickly than the low-voltage ones. Therefore, it is a faster response device than the traditional ones.

\section{CONCLUSIONS}

With the advantages of the PZT, a high-voltage piezoelectric converter was designed for applications to improve electronic safety in this paper. The proposed scenario includes the inverter drive circuit, a Rosen PZT, the output circuit, and the feedback control circuit.

The innovation of this design is to combine the PZT with a high-voltage capacitor to charge loads with high-efficiency in safety-related electronic systems. The components of the high-voltage PZT are analyzed and designed in detail. In

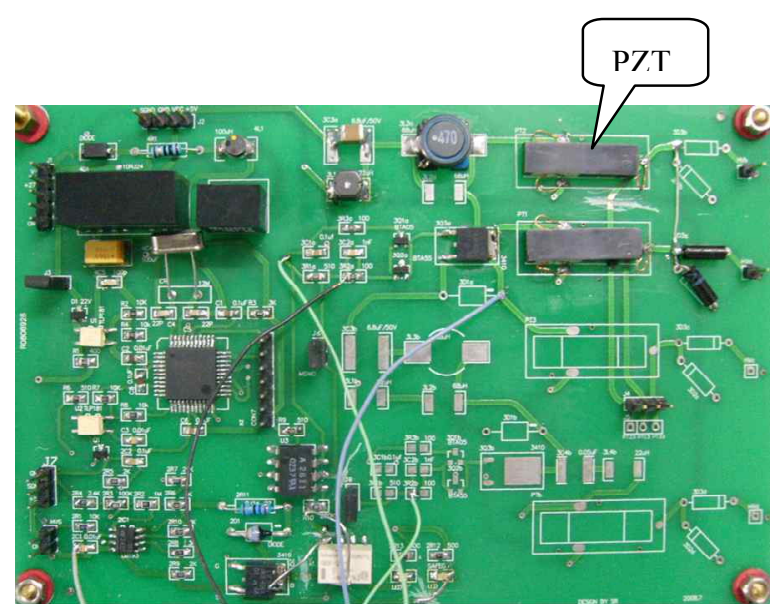

Fig.20. A test PCB of PZT convertor.

addition, the implementation of the high-voltage piezoelectric converter system is given. In order to verify the validity of the converter circuits, a prototype was developed. The experimental results are fully in line with the calculation and analysis results. The output voltage of this converter is gained and controlled in a range from $2.9 \mathrm{kV}$ to $3.1 \mathrm{kV}$ in less than $800 \mathrm{~ms}$, which works in the high-efficiency CCC mode with the ZVS condition.

In general, the advantages of the proposed system are its smaller size and lighter weight. Furthermore, through the ignition energy from $2.9 \mathrm{kV}$ to $3.1 \mathrm{kV}$ in high efficiency mode, higher reliability and accuracy are gained. Finally, the charging speed is controlled in less than $800 \mathrm{~ms}$ which means that the proposed system has a faster response time when compared with the traditional ones. With the increased ignition energy produced by the PZT converter, the reliable ignition of airbags is realized in electronic safety systems for intermediate or advanced grade vehicles.

\section{ACKNOWLEDGMENT}

This work was supported by the Open Fund of State Key Laboratory of Explosion Science and Technology from Beijing Institute of Technology (No.KFJJ10-5M), the Fund of Beijing Education Committee (No.19000530871) and Shandong University research Start-up Fund (No.1050513200003).

\section{REFERENCES}

[1] G.-B. Chung and K. D.T. Ngo, "Analysis of an AC/DC resonant pulse power converter for energy harvesting using a micro piezoelectric device," Journal of Power Electronics, Vol. 5, No. 4, pp.247-256, Oct. 2005.

[2] M. Ryu, S. Choi, S. Lee, and B. H. Cho, "A new piezoelectric transformer driving topology for universal input $\mathrm{AC} / \mathrm{DC}$ adapter using a constant frequency pwm control," in Proceeding of Applied Power Electronics Conference and Exposition(APEC), pp.1314-1317, 2006.

[3] E. Dallago, A. Danioni, G. Ricotti, and G. Venchi, "Single chip, low supply voltage piezoelectric transformer 
controller," European Solid-State Circuits, ESSCIRC Conference, pp. 273-276, 2003.

[4] J. R. Li, "Study on admittance circle diagram of piezoelectric ultrasonic wave transducer," Instrumentation Technology, Vol. 11, pp. 62-64, 2007.

[5] F. Nuno, F. J. A. Martin, J. Diaz, M. J. Prieto, and F. M. Fernandez-Linera, "Quantum mode control for piezoelectric transformers in AC/DC applications," Power Electronics Congress, Technical Proceedings, pp. 202-207, 2002.

[6] Y. Ishizuka, K.-W. Lee, T. Oyama, H. Matsuo, and T. Koga, "Consideration of a single-switch inverter for piezoelectric transformer with a new control method," PESC, IEEE 34th Annual Conference, pp. 1621-1626, 2003.

[7] G. Ivensky, I. Zafrany, and S. Ben-Yaakov, "Generic operational characteristics of piezoelectric transformers," IEEE Trans. Power Electron., Vol. 17, No. 6, pp. 1049-1057, Nov. 2002.

[8] G. Ivensky, M. Shvartsas, and S. Ben-Yaakov, "Analysis and modeling of a voltage doubler rectifier fed by a piezoelectric transformer," IEEE Trans. Power Electron., No.19, pp.542-549, Mar. 2004.

[9] T. M. Wang, et. al., MATLAB Electric Power System Design and Analysis, chap. 5, Defense Industry Publishing House, Peking, 2010.

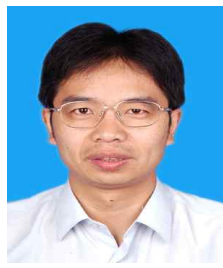

Hongbing Xiao was born in Ningxia, China, in 1968. He received his B.S. degree in Automatic Instrumentation from the Huazhong University of Science and Technology, Wuhan, China, in 1991, his M.S. degree in Mechatronics Engineering from Beijing University, Beijing, China, in 2004, and his Ph.D. degree in Mechatronics Engineering from the Beijing Institute of Technology, Beijing, China, in 2008. Since 2008, he has been working as an Associate Professor in the Computer and Information Engineering College, Beijing Technology and Business University, Beijing, China. He was a visiting scholar at the Florida International University, Miami, FL, USA. His current research interests include power electronic circuits and embedded system applications. He has published 3 books and over 30 papers.

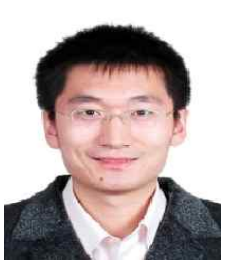

Yu Du received his B.S. degree in Electronic Science and Technology from the Wuhan University of Technology, Wuhan, China, in 2004, and his M.S. and Ph.D. degrees in Electrical Engineering from the Florida International University, Miami, FL, USA, in 2007 and 2012, respectively. Since 2013, he has been working as a Lecturer in the School of Mechanical, Electrical and Information Engineering, Shandong University, Weihai, China. His current research interests include wireless communication and power electronics.

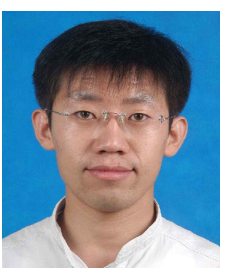

Chunyu Bai was born in Shuozhou, China, in 1981. He received his B.S. degree in Electrical Engineering from the Shanxi Agricultural University, Shanxi, China, in 2003, and his Ph.D. degree in Mechatronics Engineering from the Beijing Institute of Technology, Beijing, China, in 2009. He mainly studied piezoelectric transformers and associated power electronic circuits design while pursuing his $\mathrm{Ph} . \mathrm{D}$. Since 2009, he has been working for Changjiangyuan Science \& Technology Co., Ltd. as R\&D Senior Engineer of piezoelectric transducers and associated power electronic circuits used in high-intensity focused ultrasound systems.

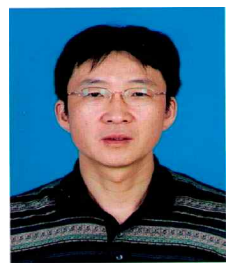

Zerong Guo received his $\mathrm{Ph} . \mathrm{D}$. degree in 2004 and works in School of Mechatronical Engineering, Beijing Institute of Technology, Beijing, China. His current research interests include the explosion hazard parameters perception, prevention and control technology, wireless sensor network tests, safety and reliability. He has published more than 30 papers and has five invention patents.

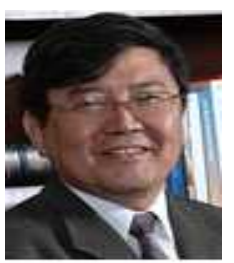

Kang K. Yen (SM'85) received his M.S. degree from the University of Virginia, Charlottesville, VA, USA, in 1979, and his $\mathrm{Ph} . \mathrm{D}$. degree from Vanderbilt University, Nashville, TN, USA, in 1985. He is currently a Professor in the Electrical Engineering Department, Florida International University, Miami, FL, USA. He is also a registered professional engineer in the state of Florida. He has been involved in theoretical work on control theory and on parallel simulation algorithms developed for real-time applications in the past several years. During this same period, he also participated in several industry supported projects on real-time data processing and microprocessor-based control system designs. His current research interests include power electronics, electronics security related issues and the performance improvement of systems. He has published over 100 papers. He has been a Chair of the Miami section of the IEEE several times and has been the Section Chair of several IEEE meetings. He has alos received awards from the IEEE including the IEEE the Third Millennium Medal, 2000, and the IEEE Service Award, Florida Council, 1993. 